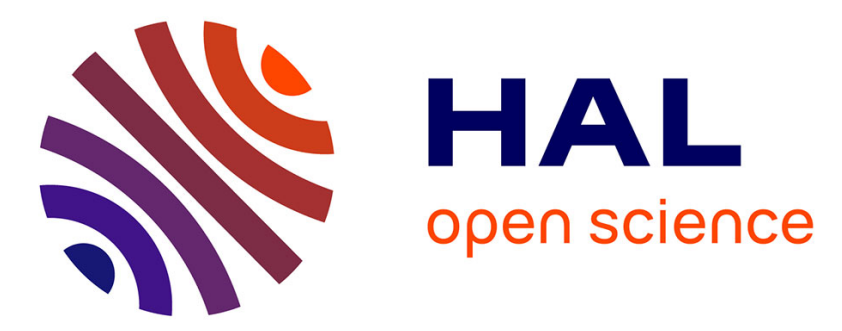

\title{
Maximizing biogas production from the anaerobic digestion
}

\author{
Amel Ghouali, Tewfik Sari, Jérome Harmand
}

\section{To cite this version:}

Amel Ghouali, Tewfik Sari, Jérome Harmand. Maximizing biogas production from the anaerobic digestion. Journal of Process Control, 2015, 36, pp.79-88. 10.1016/j.jprocont.2015.09.007 . hal01205935

\section{HAL Id: hal-01205935 \\ https://hal.science/hal-01205935}

Submitted on 28 Sep 2015

HAL is a multi-disciplinary open access archive for the deposit and dissemination of scientific research documents, whether they are published or not. The documents may come from teaching and research institutions in France or abroad, or from public or private research centers.
L'archive ouverte pluridisciplinaire HAL, est destinée au dépôt et à la diffusion de documents scientifiques de niveau recherche, publiés ou non, émanant des établissements d'enseignement et de recherche français ou étrangers, des laboratoires publics ou privés. 


\title{
Maximizing biogas production from the anaerobic digestion
}

\author{
A. Ghouali ${ }^{a, d}$, T. Sari ${ }^{b, e}$, J. Harmand, ${ }^{c, d, *}$ \\ ${ }^{a}$ Université de Tlemcen, Laboratoire d'Automatique, B.P 119 Tlemcen, Algérie \\ ${ }^{b}$ Irstea, UMR Itap, 361 rue Jean-François Breton, 34196 Montpellier France \\ ${ }^{c}$ INRA, UR0050 LBE, Avenue des Etangs, 11100 Narbonne, France \\ ${ }^{d}$ INRA-INRIA, EPI MODEMIC, UMR MISTEA, \\ 2 P. Viala, 34060 Montpellier, France \\ ${ }^{e}$ Université de Haute Alsace, 4 rue des Frères Lumière, 68093 Mulhouse, France \\ * Corresponding author.
}

17 juillet 2015

\begin{abstract}
This paper presents an optimal control law policy for maximizing biogas production of anaerobic digesters. In particular, using a simple model of the anaerobic digestion process, we derive a control law to maximize the biogas production over a period $T$ using the dilution rate as the control variable. Depending on initial conditions and constraints on the actuator (the dilution rate $D($.$) ), the search for$ a solution to the optimal control problem reveals very different levels of difficulty. In the present paper, we consider that there are no severe constraints on the actuator. In particular, the interval in which the input flow rate lives includes the value which allows the biogas to be maximized at equilibrium. For this case, we solve the optimal control problem using classical tools of differential equations analysis. Numerical simulations illustrate the robustness of the control law with respect to several parameters, notably with respect to initial conditions. We use these results to show that the heuristic control law proposed by Steyer et al., 1999 [20] is optimal in a certain sense. The optimal trajectories are then compared with those given by a purely numerical optimal control solver (i.e. the "BOCOP" toolkit) which is an open-source toolbox for solving optimal control problems. When the exact analytical solution to the optimal control problem cannot be found, we suggest that such numerical tool can be used to intuiter optimal solutions.
\end{abstract}

Keywords : optimal control, bioreactor, maximization, biogas, anaerobic digestion. 


\section{Introduction}

Anaerobic digestion or methanization is a biological process in which organic compounds are transformed into carbon dioxide and methane (biogas) by micro organisms. These processes represent a promising technology for treating liquid and solid waste while producing valuable energy and limiting the greenhouse [14]. The operation of such process poses however a number of practical problems since anaerobic digestion is a complex nonlinear system which is known to be unstable : an organic overload can destabilize the biological process and its restart needs long delays (over months). It is thus necessary to develop automatic systems to optimally manage such a process when dealing with disturbances or to optimize important steps as its operation during the starting period.

Schematically, there are two families of automatic controllers developed for such purpose : model- and knowledge-based approaches. The first refer to the synthesis and the application of automatic control laws when a model of the system is available. Most of such controllers have been proposed by people from the automatic control community. They include approaches based on both linear or nonlinear techniques. Their main advantage lie in their theoretical properties they are suppose to guarantee such as performance or stability robustness with respect to uncertainty or disturbances. For instance, the controllers proposed by [1] are part of this family. The second class of approaches - called here knowledge-based - include approaches - not only but rather - developed by experts of the anaerobic digestion (biotechnologists, chemical engineers) who proposed control methods based on the detailed practical knowledge they have about bioprocess dynamics. They are most often validated on real processes than model-based controllers and are usually based on easily accessible measurements usually available in an industrial context such as $\mathrm{pH}$, gas flow rates, H2 concentration, partial or total alkalinity... Approaches by [10], [15] or [20] can be classified in this family. The counterpart of the fact they are easily applicable on real processes is that no theoretical guarantee with respect to performance can be given since no model is necessary to synthesize the controller.

Steyer et al., 1999 [20] proposed the 'Disturbances Monitoring' principle as a way to control highly loaded anaerobic processes. This strategy is based on the following idea : a known overload is first applied voluntary to the process. The analysis of the system's response allows one to decide whether the system is able to deal with an increase in the pollutant load or not. If it is the case, the feeding flow undergoes a step while it remains at its actual value or is decreased otherwise. Once the new equilibrium has been reached, a new load test is applied and so on. In their paper, the authors claim that 'the control law allows to reach automatically the maximum treatment ability of the anaerobic process whatever the input concentration in organic matter is' without being able to prove it because their control law is completely heuristic. If we define the maximum treatment ability of an anaerobic system as the maximum biogas flow rate it can deliver over a given period of time, and if we consider a model of the process, we face an optimal control problem that can be posed from a mathematical viewpoint. To do so, we need a model of the anaero- 
bic digestion process. The anaerobic process involves thousands of microorganisms interacting together through a complex metabolic network which is, actually, only partially known. On the one hand, even if several synthetic functional models have recently been proposed, including high dimensional ones like the ADM1 (cf. [9]), but also more simplified ones like the AM2 (cf. [3]), it is to be noticed that the nonlinear character of biological models and their relatively high dimension (the AM2 is of dimension 4 in its simplest form) render their use inappropriate for the application of optimal control theory. On the other hand, it has been shown that under some circumstances, very simple models were able to adequately capture the main dynamical behavior of the anaerobic model [8].

The use of such a simplified model is not new and several authors have already proposed to use it for optimal control design of anaerobic digesters (cf. for instance [19]). From the seminal work by D'Ans and al. ([4]) who established the bang-bang character of a simple optimal-time control problem of the chemostat, the optimal control of bioprocesses in general, and of the anaerobic digestion in particular, has been studied over a quite long time. More particulary, an optimal control policy to avoid the failure in the digester operation and restore its normal operation or lead it to a new optimal steady state was proposed by Stamatelatou and al. [19]. It has been designed using a simplified model of anaerobic digestion to determine the optimal dilution rate as a function of time, in response to entry of inhibitors or sudden changes in the feed substrate concentration. The authors shown that there is a proportional relationship between the dilution rate and the methane production. A simpler and implementable suboptimal control law was derived by Dimitrova and Krastanov to stabilize in real time a dynamic model towards the maximum methane flow rate [7]. Their approach is however not model-based : the algorithm is presented in the form of a block-scheme to iteratively adjust the dilution rate to drive the process dynamics towards a set point, where an optimal value of the output is achieved. The main limitation in applying this approach is that the dynamics should be open-loop stable. Otherwise, a locally stabilizing controller is necessary to stabilize the equilibrium points around the optimal operating point [7]. Other extensions were derived by the same authors $[5,6]$. Sbarciog et al. $[16,17]$ have proposed a control strategy for maximizing biogas production of an anaerobic digestion system modeled by a 2-order system. The control law was synthesized by solving two optimization problems : firstly, a static optimization problem to determine the optimal operating point and, secondly, a transient optimization problem using the maximum principle of Pontryagin to find the control which will drive the system from an initial condition towards the optimal operating point while maximizing the gas flowrate. Recently, considering other sets of measurements, Sbarciog et al. [18] proposed a simple switching strategy for optimizing anaerobic digestion process. In [19], the problem of maximizing the biogas production over a given period of time has been investigated considering different possible disturbances (presence of an inhibitor or over/under-loads). The singular arcs were calculated using the Maximum Principle of Pontryagin. However, the optimal control synthesis was not given explicitly and no controllability analysis was performed. 
In the present work, considering i) a simple model of a chemostat (see equations (1) below), ii) a restricted set of initial conditions (see Hypothesis 2 below) iii) assuming the biogas produced is a linear function of the activity defined as $k \mu(s) x$ as proposed in [2] (see section (2)), we solve the problem of maximizing the biogas production over a given period of time $\max _{D(t)} \int_{t=0}^{t=T} \mu(s) x d t$ for a large class of kinetics functions (including both Monod and Haldane growth rates). These results are then used to better understand the knowledge-based controller proposed by Steyer and al.(1999).

This paper is organized as follows. In section (2) we present the class of problems we are interested in. In the next section, we establish the main results of the paper about the maximization of the output gas flow rate for the chemostat model. In section 4, we compare the control law we establish in section 3 with that one proposed by Steyer et al., 1999 [20]. In section 5, we propose to use a direct approach to intuiter the optimal trajectories for a more general class of initial conditions than those considered in previous sections before some conclusions and perspectives are drawn.

\section{Model description and control problem}

In the present work, we consider a single-step model for the anaerobic digestion process based on one biological reaction, where the organic substrate denoted by $s$ is degraded into methane biogas $\left(\mathrm{CH}_{4}\right)$ by a bacterial population $x$. We assume that the methane flow rate, $Q_{C_{4}}$, is proportional to the microbial activity as proposed in [2]. The mass balance model of the classical chemostat model is given by the following nonlinear system of ordinary differential equations :

$$
\left\{\begin{aligned}
\dot{x} & =(\mu(s)-D) x \\
\dot{s} & =D\left(s_{i n}-s\right)-\mu(s) x
\end{aligned}\right.
$$

where $x$ and $s$ denote biomass and substrate concentrations, respectively, $s_{i n}$ is the concentration of the influent substrate $s$, while $\mu(s)$ is the specific growth rate of biomass. $D \in\left[D_{\min }, D_{\max }\right]$ is the dilution rate which is considered hereafter as the control variable, $\mu$ is the specific growth rate of microorganisms. Notice that the yield coefficient $Y$ which usually appears in the chemostat model, does not appear in the equations since it is straightforward to show that the change of variable $x=X / Y$ allows us to reduce the system to (1). In the sequel, we will consider that the kinetic $\mu$, satisfies the very general property :

Hypothesis 1. $\mu(0)=0$ and $\mu(s)>0$ for all $s>0$. The function $\mu(\cdot)$ is either increasing, or there exists $\bar{s}$ such that $\mu(s)$ is increasing for $0<s<\bar{s}$ and decreasing for $s>\bar{s}$.

In this paper, we seek to maximize the biogas production over a given time interval. The total methane production over the interval $[0, T]$ can be expressed as :

$$
J(x(\cdot), s(\cdot), D(\cdot))=\int_{0}^{T} k \mu(s(t)) x(t) d t
$$


where $(x(\cdot), s(\cdot), D(\cdot))$ satisfy $(1)$. Since $k$ is constant, without loss of generality, we will consider $k=1$ in the following.

The problem of maximizing biogas production consist in maximazing the integral (2) under the constraint $D_{\min } \leq D(t) \leq D_{\max }$. As surprising as it may be, as posed hereabove in very general terms, the optimal control problem is very difficult to solve. It is why we restrict here our attention to a specific case in introducing the following additional hypothesis.

Hypothesis 2. The initial conditions of system (1) lie on the line $x_{0}+s_{0}=s_{\text {in }}$.

Notice that under Hypothesis $2, x(t)+s(t)=x_{0}+s_{0}=s_{i n}$ holds for all positive $t$. Thus, the two dimensional dynamical system (1) is reduced to the following one dimensional system :

$$
\dot{x}=\gamma(x)-D x ; \quad x(0)=x_{0}
$$

with $\gamma(x)=\mu\left(s_{i n}-x\right) x$. Notice that $\gamma(0)=0$ and $\gamma\left(s_{i n}\right)=0$. We can then rewrite the functional (2), with $k=1$, as :

$$
J(x(\cdot), D(\cdot))=\int_{0}^{T} \gamma(x(t)) d t
$$

where $(x(\cdot), D(\cdot))$ satisfy $(3)$, subject to constraint $D_{\min } \leq D(t) \leq D_{\max }$. Finally, to completely define our problem, we introduce the following hypotheses

Hypothesis 3 . There exists $\left.x_{*} \in\right] 0, s_{i n}[$ such that

$$
\begin{aligned}
& \gamma(x) \leq \gamma\left(x_{*}\right) \text { for all } x \in\left[0, s_{i n}\right] \\
& \gamma(x) \text { is increasing on }\left[0, x_{*}\right] \\
& \gamma(x) \text { is decreasing on }\left[x_{*}, s_{i n}\right]
\end{aligned}
$$

Hypothesis 4. Let $D_{*}=\mu\left(s_{i n}-x_{*}\right)$ be the control for which $x=x_{*}$ is a steady state of equation (3). We assume that $0 \leq D_{\min } \leq D_{*} \leq D_{\max }$.

Remark 1 Note that both Monod and Haldane functions (that are $\mu(s)=\mu_{\max } \frac{s}{s+k_{s}}$ and $\mu(s)=\mu_{\max } \frac{s}{s+k_{s}+\frac{s^{2}}{k_{i}}}$, respectively) verify Hypotheses 1 and 3 .

\section{Maximizing the output gas flow rate}

\subsection{Main results}

We first establish the following result (the proof is given in Appendix (A)) :

Proposition 1. Assume that Hypotheses 1-4 hold. Then the optimal control maximizing the functional (4) is given by:

$$
D(\cdot)=\left\{\begin{array}{lll}
D_{\min } & \text { if } x(t)<x_{*} \\
D_{\max } & \text { if } x(t)>x_{*} \\
D_{*} & \text { if } x(t)=x_{*}
\end{array}\right.
$$

where $D_{*}=\mu\left(s_{i n}-x_{*}\right)$ is the singular control which keeps $x(t)=x_{*}$ at the optimal steady state. 
In such a case, according to the position of $x(t)$ with respect to $x_{*}$, the digester operates either at its minimum $\left(D=D_{\text {min }}\right)$ or at its maximum capacity $\left(D=D_{\max }\right)$, until it reaches the singular $\operatorname{arc} D_{*}$ and stay at the optimal steady state $x_{*}$.

Remark 2 The bang-bang control law proposed within proposition 1 is equivalent to the fastest possible way to go towards the optimal point $x_{*}$ and stay there if $x_{*}$ is attained.

\subsection{Implementation problems and robustness properties}

\subsubsection{Implementation of the control law}

In this section, we discuss a number of questions related to the practical implementation of the optimal control law given in the previous section. The first question that may arise is related to the bang-bang character of the control. Indeed, when implementing the optimal strategy, we face the problem of simulating a dynamical system with a discontinuous second member ([11]). This problem can be solved using specific mathematical analysis tools and numerical algorithms. In practice, to apply this strategy on a real system, this problem is usually circumvented using regularization techniques. This approach consists in joining the two discontinuous arcs of the control by a continuous signal where the switch between two discontinuous values of the control is replaced by a continuous change between $x_{*}-\epsilon_{2}$ and $x_{*}+\epsilon_{1}$ (resp. $s_{*}-\epsilon_{2}$ and $s_{*}+\epsilon_{1}$ if $s$ is measured instead of $x$, cf. Figure 1 where $\epsilon_{1}$ and/or $\epsilon_{2}$ are tuning parameters as shown in Figure 1. Once regularized, one may check by simulation that the closed loop system behaves satisfactorily.
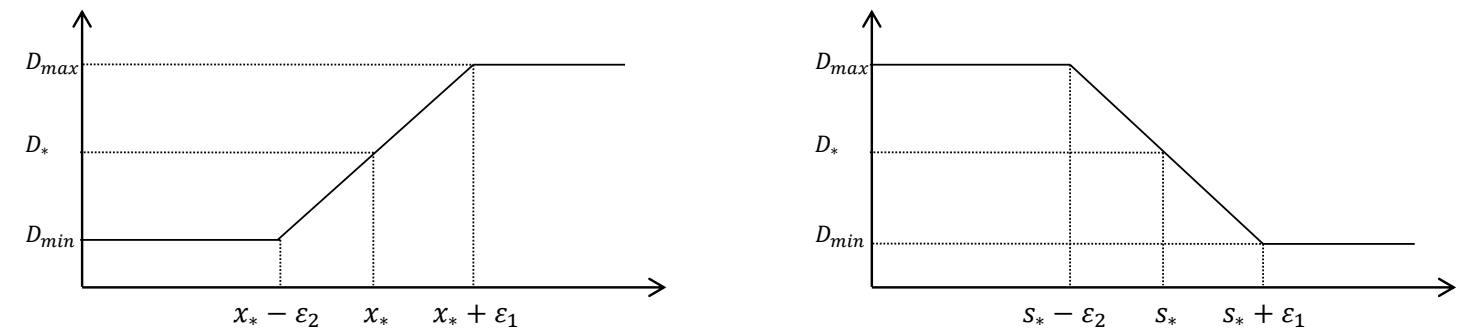

FIGURE 1 - Regularization of a discontinuous control signal depending on the measurement : $x$ is measured (left), $s$ is measured (right).

The presence of noise and the fact that sensors and/or actuators can be affected by delays may also disturb the functioning of the controller. In such cases, the use of different filters and other numerical techniques may limit the level of their influence on the closed-loop system. One must also keep in mind that measuring biological states can take some time and that actuators, like pumps in the present case, cannot deliver signals with too high derivatives. Thus, the discrete character 
of actuators and sensors must be dealt with. In more specific terms, engineers evaluate the robustness of the controller in the presence of such disturbances and if the simulation results are appropriate, do not implement sophisticated techniques. Apart from very specific application domains like in aeronautic or astronautic, such questions for biological processes are usually only studied in simulation or explored for very particular cases without considering any optimization criterion (cf. for instance [13]). However, it may happen that apparent small changes lead to important consequences for the closed-loop system. In our case, as already stated hereabove, notice that it may happen that the variable $s$ is measured instead of $x$. In such a case, a corollary of Proposition 1 can directly be written and yields the same optimal control law unlike the decision is inverted with respect to the position of $s$ with respect to $s_{*}$ since $x(t)=s_{\text {in }}-s(t)$. It seems that nothing is fundamentally changed in the control unless it is now the corollary to Proposition 1 which must be used to compute the actual control. However, if we investigate now the robustness properties of the control system, we will establish that under some circumstances, fundamental and actually dramatic changes may arise.

\subsubsection{Robustness with respect to initial conditions}

In practice, the condition $x_{0}+s_{0}=s_{i n}$, defined as the hypothesis 2 , may not be satisfied. Studying the robustness of the control with respect to the initial conditions refers to studying what happens to the system when the closed loop system is simulated with the control law synthesized in proposition 1 - that has been designed for $x_{0}+s_{0}=s_{i n}$ - while it is actually not the case.

Remark 3 When Hypothesis 2 is not satisfied, the control law is not optimal. More precisely, notice that the strategy proposed in Proposition 1 consists in applying either $D_{\min }$ or $D_{\max }$ and then to switch to the singular control $D_{*}$ which shall maintain the state on the singular arc $x=x_{*}$ or $s=s_{*}$ once it is attained. However, it has been established by Stamatelalou et al. (cf. [19]) that $s=s_{*}\left(\right.$ or $x=x_{*}$ ) are not singular arcs in the general case (however, as recalled in the introduction, in their paper Stamatelatou et al. did not give the optimal synthesis. The problem is difficult and actually remains open). Thus, the control proposed in Proposition 1 is not optimal when Hypothesis 2 is not satisfied.

First, assume $x$ is measured. Let us apply the control law (8) given in Proposition 1 for different values of $s_{0}, x_{0}$ and $s_{i n}$ such that $x_{0}+s_{0}=s_{i n}$ is no longer satisfied. To illustrate our discussion, let consider a model with Monod kinetic where $\mu_{\max }=4.5$, $K_{S}=10, S_{i n}=100, D_{\max }=1.2 D_{*}\left(\right.$ all in arbitrary appropriate units $\left.{ }^{1}\right)$. In such a case $x_{*}$ which only depends on $\mu_{\max }, K_{S}$ and $s_{\text {in }}$ equals 76.8 . Let us simulate the control over a period of time $T=2$ for several sets of initial conditions and two different values of $D_{\text {min }}$, that are $D_{\text {min }}=0$ and $D_{\min }=1$. To avoid numerical problems due to the presence of a discontinuous second member, we regularize the control law with $\epsilon_{1}=1$ yielding $\epsilon_{2}=5$ where $\epsilon_{1}$ and $\epsilon_{2}$ are defined in Figure 1 and

1. The values for $\mu_{\max }$ and $K_{S}$ will be the same in all simulations of the paper 
thus computed as :

$$
\epsilon_{2}=\epsilon_{1} \frac{D_{*}-D_{\min }}{D_{\max }-D_{*}}
$$

if $x$ is measured and as :

$$
\epsilon_{2}=\epsilon_{1} \frac{D_{*}-D_{\max }}{D_{\min }-D_{*}}
$$

if $s$ is measured. With the regulation of the control law, the system to be simulated is a simple system of ordinary differential equations which were solved using standard matlab solvers. The total biogas produced for each case is reported in the third column of Table (1) while the dynamic behavior of some interesting cases (solutions with initial conditions $\left\{s_{0}, x_{0}\right\}$ corresponding to $\{10,10\},\{10,40\}$ and $\left.\{10,70\}, D_{\min }=0\right)$ are plotted in Figure 2 .
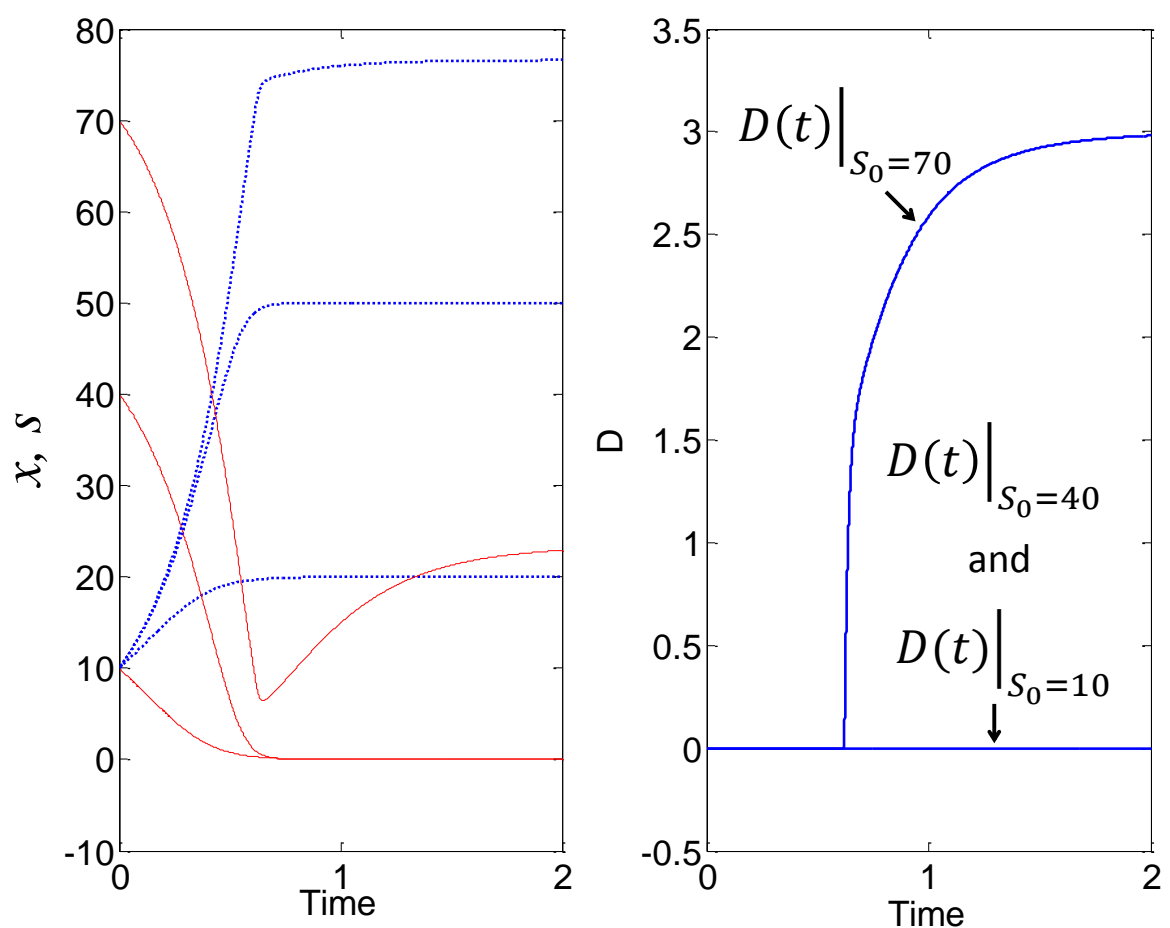

FIgURE 2 - Robustness of the optimal control when $x$ is measured and $s_{0}+x_{0}=s_{\text {in }}$ does not hold, $D_{\min }=0$ and $D_{\max }=1.2 D_{*}$; on the left, $x$ is plotted in thick dotted lines and $s$ in fine solid lines.

For several initial conditions $(\{10,10\},\{10,40\}$ and $\{50,10\}$ in Table (1)), when $x$ is measured, the system behaves as a batch and the optimal steady state is never reached even for large $T$ : since the yield of biogas production equals 1 , the total biogas produced is simply equal to $s_{0}$. The reason explaining this batch behavior is the following : as stated in Proposition 1, as long as $x_{0}<x_{*}$, the control applied is $D=D_{\text {min }}=0$ until $x(t)$ reaches $x_{*}$. If $T$ is large enough, the condition $x_{0}+s_{0}=s_{\text {in }}$ guarantees that there is enough substrate for the biomass to reach $x_{*}$. However, when $x_{0}+s_{0}=s_{i n}$ no longer holds, like in the cases where initial conditions are $\{10,10\},\{10,40\}$ and $\{50,10\}$, it may happen that there is not enough substrate for 


\begin{tabular}{|c|c|c|c|}
\hline$D_{\min }$ & $\left\{s_{0}, x_{0}\right\}$ & $\begin{array}{c}\text { functional (2) } \\
\text { when measuring } x\end{array}$ & $\begin{array}{c}\text { functional (2) } \\
\text { when measuring } s\end{array}$ \\
\hline 0 & 10,10 & 10 & 315 \\
\hline 1 & 10,10 & 239 & 320 \\
\hline 0 & 10,40 & 40 & 350 \\
\hline 1 & 10,40 & 307 & 354 \\
\hline 0 & 10,70 & 345 & 394 \\
\hline 1 & 10,70 & 341 & 384 \\
\hline 0 & 10,100 & 388 & 435 \\
\hline 1 & 10,100 & 350 & 410 \\
\hline 0 & 50,10 & 10 & 436 \\
\hline 1 & 50,10 & 344 & 437 \\
\hline 0 & 80,10 & 449 & 472 \\
\hline 1 & 80,10 & 449 & 473 \\
\hline 0 & 100,10 & 465 & 489 \\
\hline 1 & 100,10 & 465 & 490 \\
\hline
\end{tabular}

TABLE 1 - Gas flow rate wrt initial conditions, $D_{\min }$ and the measurement used. The third [resp. fourth] column gives the value of the functional (2), where $x(\cdot), s(\cdot)$ is the solution of (1) with initial condition $\left(s_{0}, x_{0}\right)$ and $D(\cdot)$ is given by $(8)$ [resp. $(9)$.

the biomass to grow sufficiently to attain $x_{*}$. In other terms, if $x_{0}+s_{0}=s_{\text {in }}$ is not verified, if $x_{0}<x_{*}$ and if $s_{0}$ is too small, then the control never switches to $D_{\max }$ and remains to $D=D_{\text {min }}=0$ leading to the production of a very small amount of biogas. The control law is thus poorly robust with respect to initial conditions when $x$ is measured.

Now, assume that $s$ is measured instead of $x$. The control law (8) given in Proposition 1 is written now

$$
D(\cdot)=\left\{\begin{array}{lll}
D_{\min } & \text { if } s(t)>s_{*} \\
D_{\max } & \text { if } s(t)<s_{*} \\
D_{*} & \text { if } s(t)=s_{*}
\end{array}\right.
$$

where $s_{*}=s_{i n}-x_{*}$.

Let us study by simulation the robustness of the control law with respect to the initial conditions. We choose $\epsilon_{1}=1$ yielding now $\epsilon_{2}=0.2$. Again, we simulate the system for the set of initial conditions reported in Table (1) and for two values of $D_{\text {min }}$. The total biogas produced for each case is reported in the fourth column of Table (1). As in the previous case, a number of simulation results are reported in Figure 3 (solutions with initial conditions $\left\{s_{0}, x_{0}\right\}$ corresponding to $\{10,10\},\{10,40\}$ and $\left.\{10,70\}, D_{\min }=0\right)$ and the total biogas produced in each case is reported in Table (1). In this case, although $s_{0}+x_{0}=s_{i n}$ is not satisfied, the system behaves well and as long as $T$ is large enough and $x_{0}>0$, the optimal point is always attained. 
Of course, the control law cannot be said to be 'optimal' with respect to the biogas production since some hypotheses are not verified, but at least, the system converges - asymptotically - towards the optimal point computed with $x_{0}+s_{0}=s_{i n}$. In this sense, we can then say that the control is robust with respect to initial conditions if $s$ is measured instead of $x$.

Furthermore, as long as $x_{*}$ is attained (i.e. $D_{\min }>0$ and $T$ large enough) we noticed that the total biogas produced is always greater when $s$ is measured instead of $x$ (compare third and fourth columns of Table 1). However, we could not prove this point and there is thus no reason to affirm that it is a general result as long as Hypothesis 2 is not satisfied.
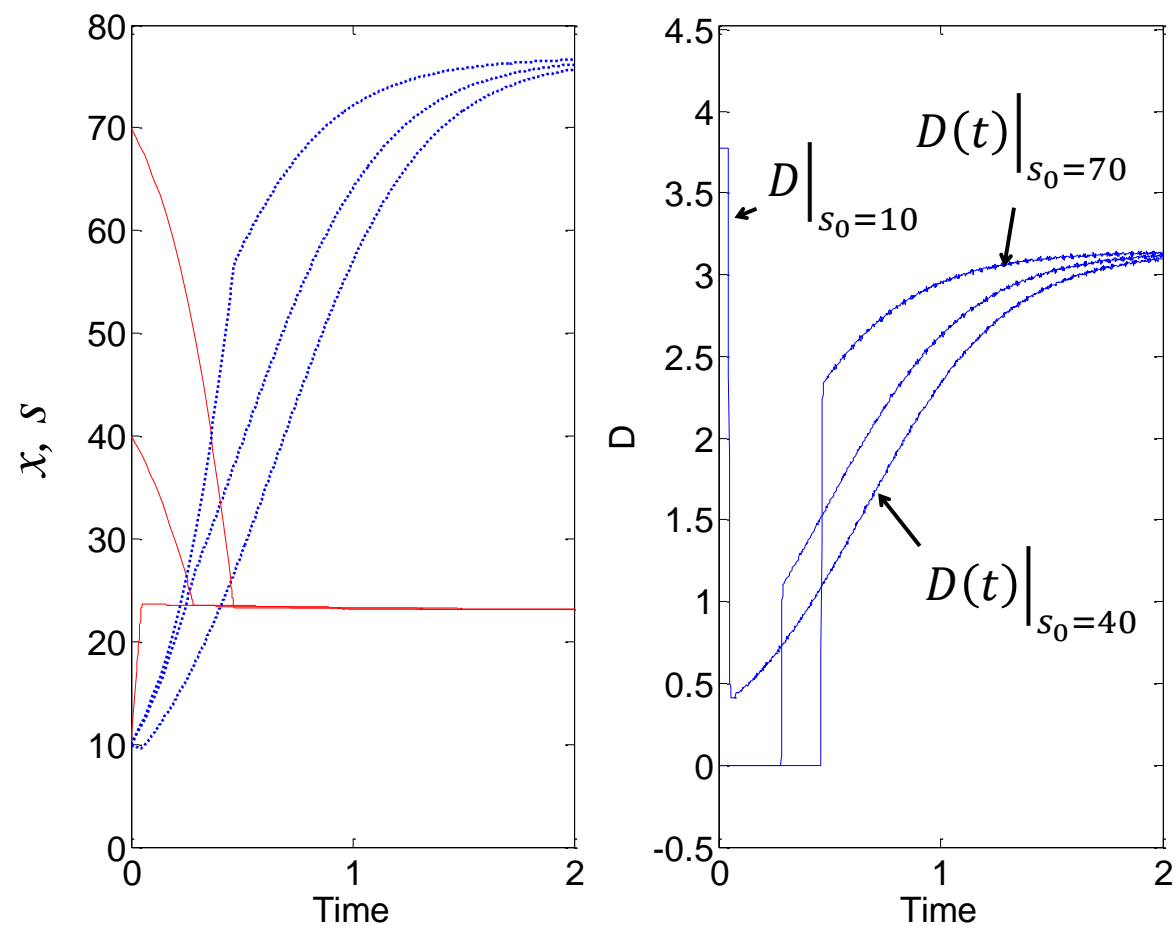

Figure 3 - Robustness of the optimal control when $s$ is measured and $s_{0}+x_{0}=s_{\text {in }}$ does not hold, $D_{\min }=0$ and $D_{\max }=1.2 D_{*}$; on the left, $x$ is plotted in thick dotted lines and $s$ in fine solid lines.

\section{Comparison with the heuristic control law}

In this section, we use the results established in Proposition 1 to show that the control law proposed in [20] and ours drive the system towards the same functioning point.

Basically, the control by [20] is based upon a test about the actual 'biological state' of the digester. The authors even presented their control law as a 'real dialog' between the operator and the microbial ecosystem of the process. The idea relies 
on the application of a controlled disturbance in the input flow rate during a given period of time. Assuming the input substrate concentration is known and constant, one may compute the theoretical increase in the gas flow this disturbance should cause. Monitoring the gas flow over the time allows then the user to estimate whether the applied overload has been supported by the process - through a corresponding increase in the biogas - or not. If yes, the process is considered to be in 'good health' and the input load can be increased through an increase of the input flow rate. If not, it means that the additional substrate that has been introduced through the disturbance has accumulated in the process (under the form of intermediate byproducts of the anaerobic digestion like VFA) possibly leading to a destabilization of the process. In such a case, the input flow rate is decreased.

Let us compare this strategy with ours. Consider the output gas flow rate represented by the $\gamma$ function in Figure 4 for the same model than in section (3.2.2) and $S_{i n}=100$. Under the hypothesis that $x_{0}+s_{0}=s_{i n}$, the dynamic of the system is given by $\dot{x}=\gamma(x)-D x$. First, consider an equilibrium point $\bar{x}$ such that $\bar{x}<x_{*}$ (left side of Figure 4). Notice that the corresponding input flow rate verifies $D_{\text {max }}>\bar{D}=\mu\left(s_{i n}-\bar{x}\right)>D_{*}$. In this case, we know that the optimal control consists in applying $D=0$ and that this control will drive the system towards $x_{*}=76.8$ (once attained, the control is then switched to $D_{*}$ ). Now, from the same initial equilibrium point $\bar{x}<x_{*}$, let us apply the control by Steyer et al., 1999. In particular, let us apply a disturbance $D_{d}>\bar{D}$ during a given period of time (step 1 on the left in Figure 4) and let us monitor the output of the system, that is the output gas flow rate. Since the dynamic of the system is given by $\dot{x}=\gamma(x)-D_{d} x, x$ will decrease (step 2 on the left in Figure 4) and since $\gamma$ is increasing for all $x<x_{*}$, we will observe a decrease in the biogas. Following the algorithm of Steyer et al., 1999 [20], we should conclude that the system does not behave very well and decrease the value of $\bar{D}$ accordingly (step 3 on the left in Figure 4). Reproducing again the same strategy, it is quite clear, at least intuitively, that the applied control should converge towards $D_{*}$, which is actually the optimal strategy since it will finally drive the state $x$ towards $x_{*}$. Conversely, if we start from an initial condition with a $\bar{D}<D_{*}$ such that $\bar{x}>x_{*}$, we can show using the same reasoning than previously (and taking into account that $\gamma$ is decreasing for $x>x_{*}$ ), that the heuristic control will drive the state towards $x_{*}$ as the optimal control (steps 1 to 3 on the right in Figure 4). Obviously, the heuristic strategy is not optimal with respect to the functional (4) since it will take more time to reach the optimal state $x_{*}$ than using the bang-bang optimal strategy, but we can still conclude that the control is similar to ours in the sense it will drive the system towards the optimal point $x_{*}$ which maximizes the output gas flow rate.

\section{A direct approach}

In previous sections, we have designed an optimal control law for the system (1) with respect to the functional (4) under Hypotheses 1 to 3. Using tools of the optimal control theory, under Hypothesis 2, we were able to find the optimal feedback law to 


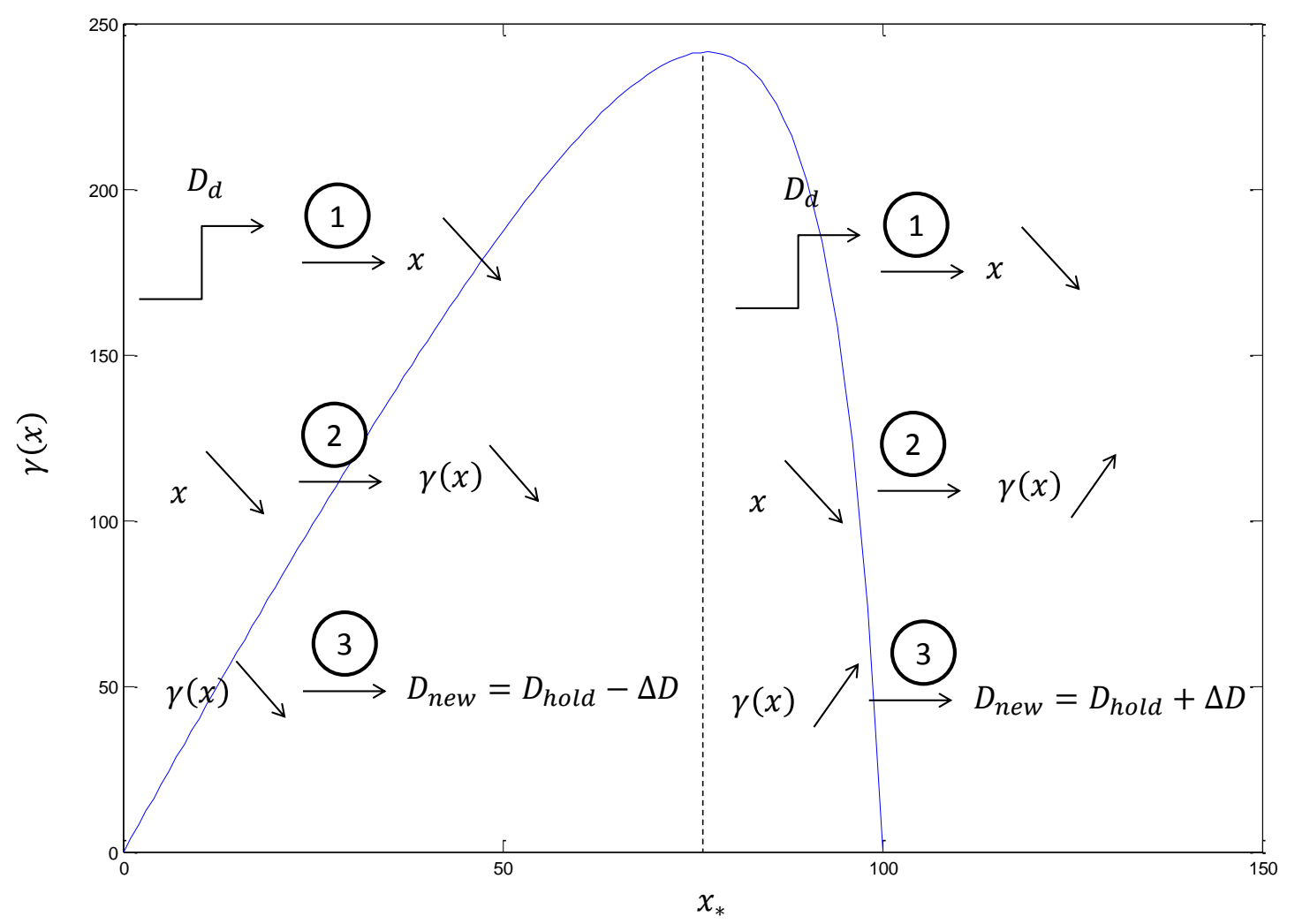

Figure 4 - The function $\gamma$ and the heuristic control law.

be applied in measuring one state of the system. When Hypothesis 2 does not hold anymore or when the model of the system is more complicated than ours so as it is not possible anymore to derive analytically the solution, a direct approach can be used. Here a 'direct approach' is referred to as a completely numerical optimization. The last decade, a number of softwares have been designed for that purpose. Here, we use a software called BOCOP ([12]). The principle of this tool is to discretize the problem and to find optimal trajectories among all candidates using numerical optimization toolkits. We have used this software in a 'blind way', i.e. without introducing any other knowledge than the criterion to be optimized, the model of the system and the known constraints on the actuator.

First, let us compare what BOCOP delivers when Hypothesis 2 holds with the application of the exact optimal solution. Again, we use the same parameters as those used in the section (3.2.2). In this case, recall that $x_{*}=76.87$ and $D_{*}=3.14$. The exact optimal control is given by Proposition 1 as long as Hypotheses 1 to 3 hold. Let $x_{0}=10$ and $s_{0}=90$. Again, to avoid numerical problems, the control is regularized with $\epsilon_{1}=1$. Let us compare the trajectories obtained when applying Proposition 1 and those computed by BOCOP for this first example. Both state trajectories and corresponding control signals are plotted in Figure 5 while BOCOP 
optimization parameters and results are reported in Appendix (B).
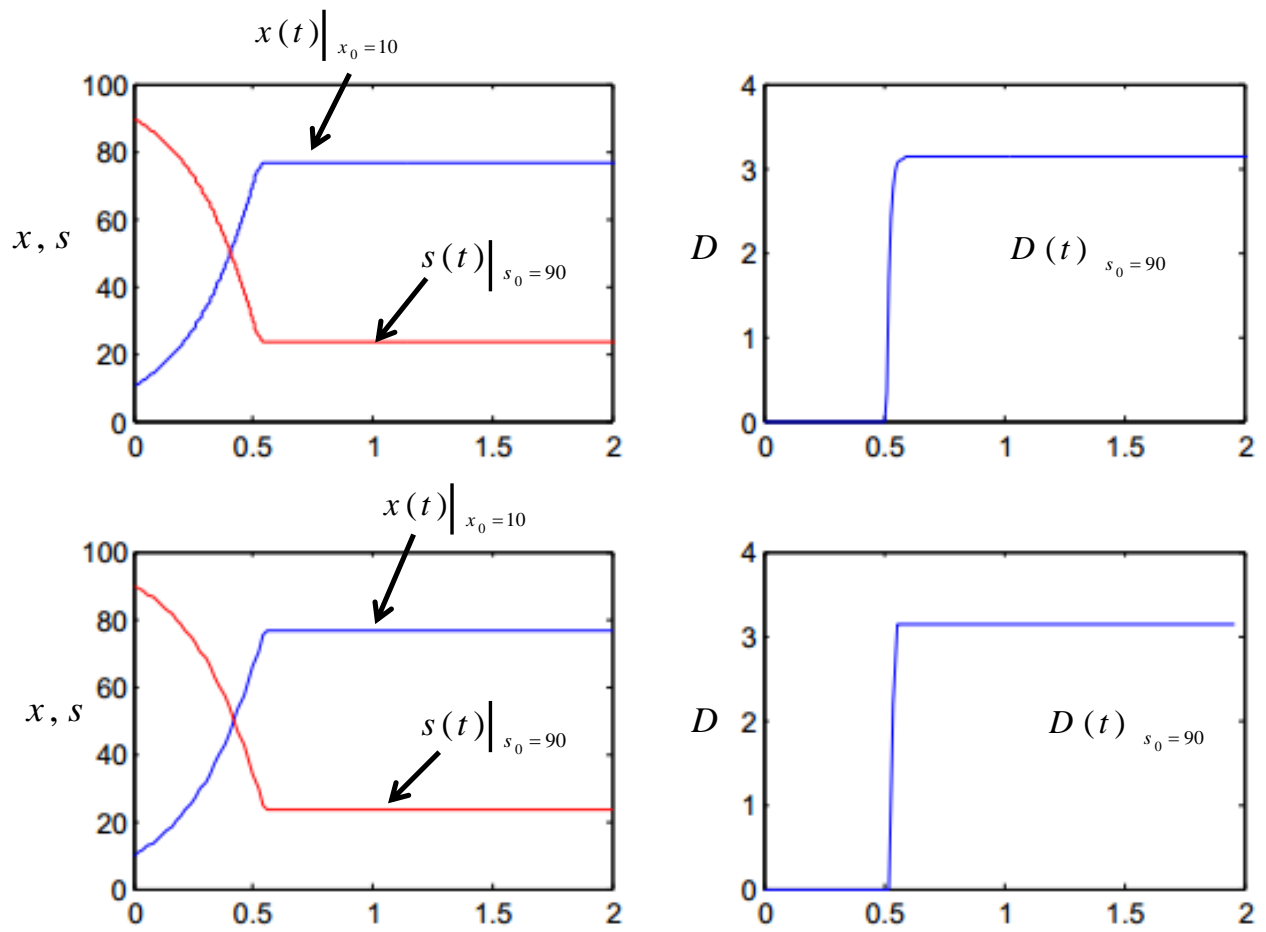

Figure 5 - Exact optimal control and optimal control computed with BOCOP : Monod kinetic, $s_{0}+x_{0}=s_{i n}$.

The flowrates in both cases are equivalent (equal to about 422). Obviously, BOCOP delivers a control signal which is comparable to the theoretical optimal solution.

These results suggest that BOCOP may be a good candidate to investigate extensions of the optimal control solutions when hypotheses used in the design of the optimal control do not hold anymore. In particular, we can investigate what could be the optimal controls computed with BOCOP when $s_{0}+x_{0}=s_{\text {in }}$ does not hold. Thus, one may compare the results obtained in the section (3.2.1) within the framework of the study of the robustness of the control law with respect to the initial conditions and those obtained with BOCOP. For that purpose, we solve the problem with BOCOP using $x_{0}=10$ and $s_{0}=10,40$ and 70, respectively. Simulation results are presented in Figure 6 while the total biogas produced in each case is reported in Table (2) and BOCOP optimization parameters and results are reported in Appendix (B).

More precisely, three cases are represented in Figure 6. For easily comparing the trajectories obtained within the robustness study (applying Proposition 1 while 

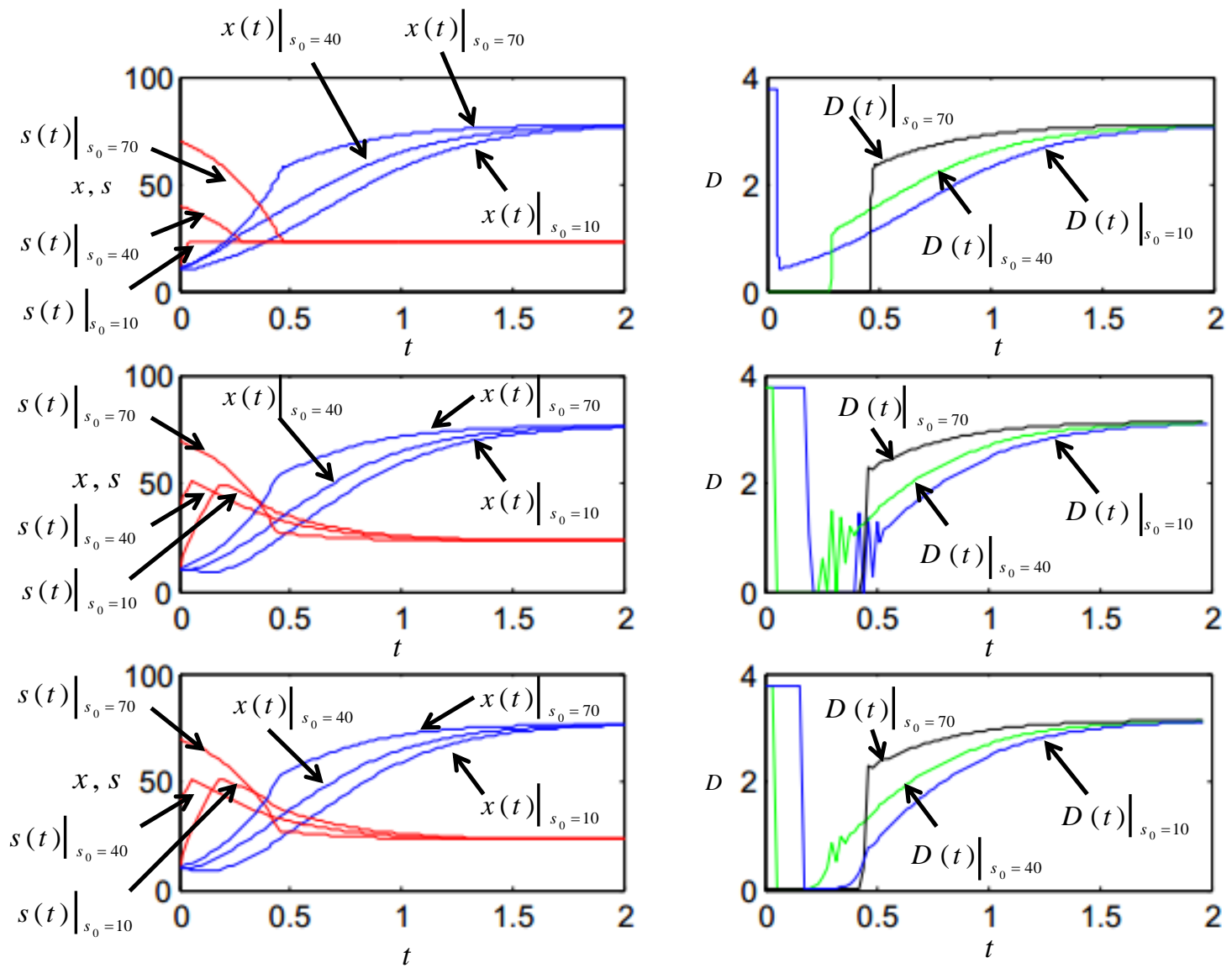

Figure 6 - Comparison of optimal controls when $s_{0}+x_{0}=s_{\text {in }}$ does not hold ; Monod kinetic and $D_{*} \leq D_{\max }$ : on the top, the plots are the same as those in Figure (3); on the medium, optimal trajectories and controls computed with BOCOP and on the bottom, the same trajectories and control when the criterion is modified in penalizing the control to avoid control chattering $(\epsilon=5)$.

$s_{0}+x_{0}=s_{\text {in }}$ does not hold) and those obtained with BOCOP, we plotted again the trajectories and controls of Figure 3 on the top of Figure 6. Just below, medium place of Figure 6, we plotted the optimal trajectories and corresponding controls obtained with BOCOP for the three initial conditions. The control signals are very noisy, simply because BOCOP has difficulties when the trajectory follows a singular arc. To avoid such chattering phenomena when using a numerical optimization software, one way to proceed is to modify the criterion to be optimized in penalizing the control signal. The criterion then becomes :

$$
J(x(.), s(.), D(.))=\int_{0}^{T}\left[\mu(s(t)) x(t)+\epsilon D(t)^{2}\right] d t
$$

Of course, when proceeding this way, a fine compromise must be found when choosing $\epsilon$ which must be kept small enough : it is expected that smaller $\epsilon$ closer the computed trajectories from the optimal ones since functional (10) tends towards 


\begin{tabular}{|c|c|}
\hline$s_{0}$ & functional $(2)$ \\
\hline 10 & 324 \\
\hline 40 & 357 \\
\hline 70 & 395 \\
\hline
\end{tabular}

TABLE 2 - Gas flow rate when computing the optimal solution with BOCOP in the case where no penalty on the control in the criterion.

\begin{tabular}{|c|c|c|}
\hline$s_{0}$ & functional (10) & functional (2) \\
\hline 10 & 388 & 324 \\
\hline 40 & 416 & 354 \\
\hline 70 & 461 & 394 \\
\hline
\end{tabular}

TABLE 3 - Functional (10) when computing the optimal solution with BOCOP in the case where a penalty on the control is added in the criterion (here for $\epsilon=5$ ) and the corresponding biogas produced (functional (2)) over the period $T=2$.

functional (2) when $\epsilon$ tends towards zero. The trajectories and controls corresponding to optimizations obtained with $\epsilon=5$ are plotted in the last line of Figure 6 (again, BOSOP optimization parameters and results are reported in Appendix (B)). To evaluate the different results, we can first compare the biogas production values reported in Table (1) of section (3.2.2) and those obtained with BOCOP (without penalty on the control) reported in Table (2) : for each pair of initial conditions considered, we notice that the total biogas produced is comparable. then, we can compare the optimal control performance with and without penalty on the control in the criterion in comparing results reported in Tables (2) and (3). Again, the results are comparable : whatever the control is left free or constrained by an additional penalty in the criterion, the optimal strategies are comparable for identical initial conditions. Thus, on the first hand, we suggest BOCOP to be a good candidate to rapidly intuiter optimal solutions when the analytical solution of a problem is not known. On the other hand, these results suggest that applying the control law proposed within the framework of Proposition 1 while Hypothesis 2 does not hold should produce reasonably good results in terms of biogas production and that the optimal solution is thus quite robust to uncertainty on the initial solutions as long as $s$ is measured.

\section{Conclusions and perspectives}

In this work, we have discussed classical implementation problems and robustness issues with respect to recent results from optimal control theory for the chemostat with the class of kinetics satisfying Hypotheses 1 and (3). Using these results, the optimality of the heuristic control law initially proposed by Steyer et al., 1999 was investigated. It was shown that this heuristic control law is suboptimal with respect to the maximization of the biogas over a given period of time and that it drives the 
system towards the same optimal point than the actual optimal control law. Perspectives of this work include the extension of our results to more complex models than the simple chemostat model and, eventually based on numerical results obtained with a numerical optimization toolkit, e.g. the BOCOP software, the generalization of the results in the case where the condition $x_{0}+s_{0}=s_{\text {in }}$ does not hold, which, actually, remains an open problem.

Acknowledgment : The authors would like to thank the Euro-Mediterranean research network TREASURE (INRA-INRIA Euromed $3+3$ project), the PHC TASSILI project no $33254 \mathrm{QH}$ and the Averroes program for their support. They also thank Terence Bayen, Claude Lobry, Ali Moussaoui and Alain Rapaport for fruitful discussions about this work.

\section{Références}

[1] V. Alcaraz-Gonzalez, A. Maloum, J. Harmand, A. Rapaport, J. P. Steyer., V. Gonzalez-Alvarez, C. Pelayo Ortiz, Robust intervalbased SISO and SIMO regulation for a class of highly uncertain bioreactors : application to the anaerobic digestion, Proceedings of the 39th IEEE CDC, Sydney, Autralia, 12-15 December (2000).

[2] G. Bastin., D. Dochain., On-line Estimation and Adaptative Control of Bioreactors, Elsevier, Amsterdam, (1990).

[3] O. Bernard., Z. Hadj-Sadok.,D. Dochain., A. Genovesi., J.P, STEYER., Dynamical model developpement and parameter identification for anaerobic wastwater treatment processl Biotechnology and Bioengineeriing, 75 (4) (2001).

[4] G. D'Ans, P. Kokotovic, D. Gottlieb, Time-Optimal Control for a Model of Bacterial Growth, Journal of optimization theory and applications, 7,1 (1971).

[5] N. Dimitrova., M. Krastanov., Nonlinear stabilizing control of an uncertain bioprocess, Int. J. Appl. Math. Comput. Sci, 19(3) :441-454, (2009).

[6] N. Dimitrova., M. Krastanov., Nonlinear adaptive control of a model of an uncertain fermentation process Int. J. Robust Nonlinear Control, 20 :1001-1009, (2010).

[7] N. Dimitrova., M. Krastanov., Nonlinear adaptative stabilizing control of an anaerobic digestion model with unknown kinetics, Int.J.Robust.Nonlinear Control, Published online in Wiley Online Library (wileyonlinelibrary.com). DOI : 10.1002/rnc.1782, (2011).

[8] C. García-Diéguez, O. Bernard, E. Roca, Reducing the Anaerobic Digestion Model No. 1 for its application to an industrial wastewater treatment plant treating winery effluent wastewater, Bioresource Technology, 132, 244-253 (2013). 
[9] IWA Task Group for Mathematical Modeling of Anaerobic Digestion Processes. Anaerobic Digestion Model No.1 (ADM1). Scientific and Technical Report No. 13 (2002), IWA Publishing, London.

[10] J. Liu, G. Olsson And B. Mattiasson, Extremum-seeking with variable gain control for intensifying biogas production in anaerobic fermentation, Water Science and Technology, $53: 4-5,35-44$ (2006).

[11] C. Lobry, T. SARI., Equations différentielles à second membres discontinus, in Contrôle non linéaire et Applications, Travaux en Cours no. 64, Hermann, Paris, 255-289, (2005).

[12] Bonnans F. J., Martinon P., Grélard V. BOCOP - A collection of examples., Research Report - http ://hal.inria.fr/hal-00726992, (2012).

[13] Mazenc, F., J. Harmand, H. Mounier, Stabilization of the chemostat with delayed sampled measurements., NOLCOS 2013, September 4-6, Toulouse, France, (2013).

[14] T. REHL, J. MüLleR CO2 abatement costs of greenhouse gas (GHG) mitigation by different biogas conversion pathways., Journal of Environmental Management, 114 :15,13-25, (2013).

[15] J. Rodryguez, G. Ruiz, F. Molina, E. Roca And J.M. Lema, A hydrogen-based variable-gain controller for anaerobic digestion processes, Water Science and Technology, 54 :2, 57-62 (2006).

[16] M. Sbarciog., M. Loccufier., A. Vande Wouwer., On the optimisation of Biogas Production in Anaerobic Digestion Systems. In preprints of the 18th IFAC World cCongress, 7150-7155, Milano Italy, (2011).

[17] M. Sbarciog., M. Loccufier., A. Vande Wouwer., An Optimizing startup strategy for a biomethanor. Bioprocess and biosystems Engineering, 35, 565-578, (2012).

[18] M. Sbarciog., J. Moreno., A. Vande Wouwer., A biogas-Based Switching Control Policy for Anaerobic Digestion Systems. Preprints of the 8th IFAC Symposium on Advanced Control of chemical Processes, (2012).

[19] G. Stamatelatou, C. Lyberatos, S. Tsiligiannis, P. Pavlou, PullamMANAPPallil, S.A. Svoronos, Optimal and suboptimal control of anaerobic digesters. Environmental Modeling and Assessment, 2 355-363 (1997).

[20] J.P. Steyer, P. Buffière, D. Rolland, R. Moletta., Advanced control of anaerobic digestion processes throug disturbances modeling. Wat. Res. Vol. 33, No. 9, pp. 2059-2068, (1999).

\section{A Proof of Proposition 1}

Let $D(\cdot)$ be an admissible control. Let $x\left(t, x_{0}, D(\cdot)\right)$ be the solution of the system :

$$
\dot{x}=\gamma(x)-D(t) x, \quad x(0)=x_{0}
$$

We need the following lemma : 
Lemma 2. Let $D=D(\cdot)$ a control satisfying $D_{\min } \leq D(t) \leq D_{\max }$ for all $t \in[0, T]$. We have :

$$
x\left(t, x_{0}, D_{\max }\right) \leq x\left(t, x_{0}, D(\cdot) \leq x\left(t, x_{0}, D_{\min }\right), \text { for all } t \in[0, T]\right.
$$

Proof. It comes from a direct application of the theorem of comparison of solutions of dynamical systems since for any control $D(\cdot)$ satisfying $D_{\min } \leq D(t) \leq D_{\max }$ one has :

$$
\gamma(x)-D_{\max } x \leq \gamma(x)-D(t) x \leq \gamma(x)-D_{\min } x
$$

In Section (A.1), we give the proof of Proposition 1 for an increasing function $\mu(\cdot)$. When $\mu(\cdot)$ is increasing and then decreasing (like a Haldane growth function) the proof is similar with some technicalities, see Section (A.2).

\section{A.1 The case of a Monod type function}

Proof. In this section, we assume that $\mu(\cdot)$ is increasing. According to Hypothesis $4, D_{\text {min }} \leq D_{*}, \mu\left(s_{*}\right)=D_{*}$ and $s_{*}<s_{i n}$. Thus there exists $s_{\text {min }}$ such that $\mu\left(s_{\text {min }}\right)=$ $D_{\min }$ and $s_{\min } \leq s_{*}<s_{\text {in }}$. Recall that $x\left(t, x_{0}, D_{\min }\right)$ is the solution of :

$$
\dot{x}=\gamma(x)-D_{\min } x, \quad x(0)=x_{0}
$$

The solution of (12) converges towards $x_{\text {min }}=s_{\text {in }}-s_{\text {min }} \geq x_{*}$. Hence for all $x_{0} \leq x_{*}$ there exists $t_{\min }\left(x_{0}\right)$ such that $x\left(t_{\min }\left(x_{0}\right), x_{0}, D_{\min }\right)=x_{*}$.

Similarly, $x\left(t, x_{0}, D_{\max }\right)$ is the solution of :

$$
\dot{x}=\gamma(x)-D_{\max } x, \quad x(0)=x_{0}
$$

If $D_{\max } \geq \sup \mu(\cdot)$, the solution of (13) converges towards 0 . If $D_{\max }<\sup \mu(\cdot)$, let $s_{\max }$ be defined by $\mu\left(s_{\max }\right)=D_{\max }$. According to Hypothesis 4 , we have $s_{*} \leq s_{\max }$. Two cases must be distinguished : If $s_{\max }<s_{\text {in }}$ then the solution of (13) converges towards $x_{\text {max }}=s_{\text {in }}-s_{\max } \leq x_{*}$. If $s_{\max } \geq s_{\text {in }}$ it converges towards 0 . Hence for all $x_{0} \geq x_{*}$ there exists $t_{\max }\left(x_{0}\right)$ such that $x\left(t_{\max }\left(x_{0}\right), x_{0}, D_{\max }\right)=x_{*}$.

Let us define

$$
t_{*}\left(x_{0}\right)=\left\{\begin{array}{lll}
\min \left(T, t_{\min }\left(x_{0}\right)\right) & \text { if } & x_{0} \leq x_{*} \\
\min \left(T, t_{\max }\left(x_{0}\right)\right) & \text { if } & x_{0} \geq x_{*}
\end{array}\right.
$$

In other words, $t_{*}\left(x_{0}\right)$ is the instant at which the solution $x\left(t, x_{0}, D_{\min }\right)$ reaches $x_{*}$, when $x_{0} \leq x_{*}$, or $x\left(t, x_{0}, D_{\max }\right)$ reaches $x_{*}$, when $x_{0} \geq x_{*}$, if its reaches it on $[0, T]$. If these solutions never reach $x_{*}$ on $[0, T], t_{*}\left(x_{0}\right)$ is put equal to $T$.

Now, to prove Proposition 1, we consider an admissible control $D(\cdot)$ and an initial condition $\left.x_{0} \in\left[0, s_{i n}\right)\right]$. To simplify notations we note $x(t)=x\left(t, x_{0}, D(\cdot)\right)$. We distinguish two cases depending on $x_{0}$.

If $0 \leq x_{0} \leq x_{*}$ then let us consider the following control :

$$
D^{*}(t)= \begin{cases}D_{\min } & \text { if } \quad 0 \leq t \leq t_{*}\left(x_{0}\right) \\ D_{*} & \text { if } \quad t_{*}\left(x_{0}\right)<t \leq T\end{cases}
$$


where $t_{*}\left(x_{0}\right)$ is defined by (14). The corresponding solution is given by

$$
x^{*}(t)= \begin{cases}x\left(t, x_{0}, D_{\min }\right) & \text { if } 0 \leq t \leq t_{*}\left(x_{0}\right) \\ x_{*} & \text { if } t_{*}\left(x_{0}\right)<t \leq T\end{cases}
$$

Using Lemma 2, for all admissible control $D(\cdot)$ one has

$$
x(t) \leq x\left(t, x_{0}, D_{\min }\right)=x^{*}(t) \text { for all } t \in\left[0, t_{*}\left(x_{0}\right)\right]
$$

Since $x^{*}(t) \leq x_{*}$ for all $t \in\left[0, t_{*}\left(x_{0}\right)\right]$, from (6) we deduce that

$$
\gamma(x(t)) \leq \gamma\left(x^{*}(t)\right) \text { for all } t \in\left[0, t_{*}\left(x_{0}\right)\right]
$$

Since $x^{*}(t)=x_{*}$ for all $t \in\left[t_{*}\left(x_{0}\right), T\right]$, from (5) we deduce that

$$
\gamma(x(t)) \leq \gamma\left(x^{*}(t)\right) \text { for all } t \in\left[t_{*}\left(x_{0}\right), T\right]
$$

Using (16) and (17), for all $t \in[0, T], \gamma(x(t)) \leq \gamma\left(x^{*}(t)\right)$. Therefore, for all $x_{0} \leq x_{*}$

$$
J(x(\cdot), D(\cdot))=\int_{0}^{T} \gamma(x(t)) d t \leq \int_{0}^{T} \gamma\left(x^{*}(t)\right) d t=J\left(x^{*}(\cdot), D^{*}(\cdot)\right)
$$

If $x_{*} \leq x_{0} \leq s_{\text {in }}$ then let us introduce the following control :

$$
D^{*}(t)= \begin{cases}D_{\max } & \text { if } 0 \leq t \leq t_{*}\left(x_{0}\right) \\ D_{*} & \text { if } \quad t_{*}\left(x_{0}\right)<t \leq T\end{cases}
$$

where $t_{*}\left(x_{0}\right)$ is defined by (14). The corresponding solution is given by

$$
x^{*}(t)= \begin{cases}x\left(t, x_{0}, D_{\max }\right) & \text { if } 0 \leq t \leq t_{*}\left(x_{0}\right) \\ x_{*} & \text { if } t_{*}\left(x_{0}\right)<t \leq T\end{cases}
$$

Using Lemma 2, for all admissible control $D(\cdot)$ one has

$$
x(t) \geq x\left(t, x_{0}, D_{\max }\right)=x^{*}(t) \text { for all } t \in\left[0, t_{*}\left(x_{0}\right)\right]
$$

Since $x^{*}(t) \geq x_{*}$ for all $t \in\left[0, t_{*}\left(x_{0}\right)\right]$, from (7) we deduce that

$$
\gamma(x(t)) \leq \gamma\left(x^{*}(t)\right) \text { for all } t \in\left[0, t_{*}\left(x_{0}\right)\right]
$$

Since $x^{*}(t)=x_{*}$ for all $t \in\left[t_{*}\left(x_{0}\right), T\right]$, from (5) we deduce that

$$
\gamma(x(t)) \leq \gamma\left(x^{*}(t)\right) \text { for all } t \in\left[t_{*}\left(x_{0}\right), T\right]
$$

Using (20) and (21), for all $t \in[0, T], \gamma(x(t)) \leq \gamma\left(x^{*}(t)\right)$. Therefore, for all $x_{0} \geq x_{*}$

$$
J(x(\cdot), D(\cdot))=\int_{0}^{T} \gamma(x(t)) d t \leq \int_{0}^{T} \gamma\left(x^{*}(t)\right) d t=J\left(x^{*}(\cdot), D^{*}(\cdot)\right)
$$

From (18) and (22) we deduce that for all initial conditions $x_{0} \in\left[0, s_{i n}\right]$ and the admissible control $D(\cdot)$ one has

$$
J\left(x^{*}(\cdot), D^{*}(\cdot)\right)=\max _{D_{\min } \leq D(\cdot) \leq D_{\max }} J(x(\cdot), D(\cdot)) .
$$

Thus, depending on the initial conditions, the control defined by (15) and by (19) is optimal, which is exactly what Proposition 1 states. 


\section{A.2 The case of a Haldane type function}

Proof. We assume that there exists $\bar{s}$ such that $\mu(s)$ is increasing for $0<s<\bar{s}$ and decreasing for $s>\bar{s}$. The numbers $s_{\min }$ or $s_{\max }$ were defined in the previous sections as solutions of equations

$$
\mu(s)=D_{\min } \text { or } \mu(s)=D_{\max }
$$

respectively. Now these equations can have at most two solutions and we must explain how to choose their appropriate solution $s_{\text {min }}$ or $s_{\text {max }}$.

Let $\left.x_{*} \in\right] 0, s_{i n}$ g given by Hypothesis 3 . Then $\gamma^{\prime}\left(x_{*}\right)=0$. Since

$$
\gamma^{\prime}(x)=\mu\left(s_{\text {in }}-x\right)-\mu^{\prime}\left(s_{i n}-x\right) x
$$

we have $\mu^{\prime}\left(s_{*}\right)=\mu^{\prime}\left(s_{i n}-x_{*}\right)>0$, that is to say $0<s_{*}<\bar{s}$.

According to Hypothesis $4, D_{\min } \leq D_{*}, \mu\left(s_{*}\right)=D_{*}$ and $s_{*}<s_{i n}$. Thus, there exists $s_{\text {min }}$ and $s_{\text {min }}^{\sharp}$ such that $\mu\left(s_{\text {min }}\right)=\mu\left(s_{\text {min }}^{\sharp}\right)=D_{\text {min }}$ and

$$
s_{\text {min }} \leq s_{*}<s_{\text {in }} \text { and } s_{\text {min }}<\bar{s}<s_{\text {min }}^{\sharp}
$$

Two cases must be distinguished : If $\mu\left(s_{i n}\right) \geq D_{\text {min }}$, that is to say $s_{i n} \leq s_{\text {min }}^{\sharp}$ then for all $x_{0} \leq x_{*}$, the solution of (12) converges towards $x_{\text {min }}=s_{\text {in }}-s_{\min } \geq x_{*}$. Therefore, there exists $t_{\min }\left(x_{0}\right)$ such that $x\left(t_{\min }\left(x_{0}\right), x_{0}, D_{\text {min }}\right)=x_{*}$. If $\mu\left(s_{i n}\right)<D_{\text {min }}$, that is to say $s_{\text {in }}>s_{\text {min }}^{\sharp}$ then, for all $x_{0} \leq x_{*}$, the solution of (12) converges either towards $x_{\text {min }} \geq x_{*}$, either towards 0 . More precisely,

1. if $x_{\text {min }}^{\sharp}<x_{0} \leq x_{*}$, where $x_{\text {min }}^{\sharp}=s_{\text {in }}-s_{\text {min }}^{\sharp}>0$, the solution of (12) converges towards $x_{\min } \geq x_{*}$, and we define $t_{\min }\left(x_{0}\right)$ by $x\left(t_{\min }\left(x_{0}\right), x_{0}, D_{\min }\right)=x_{*}$

2. if $0<x_{0}<x_{\text {min }}^{\sharp}$, the solution of (12) converges towards 0 , so that it never attains $x_{*}$, and we let $t_{\min }\left(x_{0}\right)=+\infty$.

If $D_{\max } \geq \sup \mu(\cdot)$, the solution of (13) converges towards 0 . If $D_{\max }<\sup \mu(\cdot)$, let $s_{\max }$ and $s_{\text {max }}^{\sharp}$ such that $\mu\left(s_{\max }\right)=\mu\left(s_{\max }^{\sharp}\right)=D_{\max }$ and

$$
s_{*} \leq s_{\max }<\bar{s}<s_{\max }^{\sharp}
$$

Many cases must be distinguished : If $s_{\max } \geq s_{\text {in }}$ then the solution of (13) converges towards 0 . If $s_{\max }<s_{\text {in }}<s_{\text {max }}^{\sharp}$, then it converges towards $x_{\max }=s_{\text {in }}-s_{\max } \leq x_{*}$. If $s_{\text {max }}^{\sharp}<s_{\text {in }}$, then it converges either towards $x_{\text {max }} \leq x_{*}$, either towards 0 . Therefore for all $x_{0} \geq x_{*}$, the solution attains $x_{*}$ and therefore, there exists $t_{\max }\left(x_{0}\right)$ such that $x\left(t_{\max }\left(x_{0}\right), x_{0}, D_{\max }\right)=x_{*}$.

We define now $t_{*}\left(x_{0}\right)$ by (14). The rest of the proof is the same as in Section (A.1).

Remark 4 If $\mu\left(s_{i n}\right)<D_{\text {min }}$, the washout is locally stable and there exist initial conditions (defined as $0<x_{0}<x_{\text {min }}^{\sharp}$ ) for which $x$ converges towards 0 . Notice however that it never happens if $D_{\min }=0$. 


\section{B BOCOP optimization parameters for results re- ported in section 5}

Hereafter, we report BOCOP parameters for the 7 optimizations realized with BOCOP reported in Figures 5 and 6

1. Simulations of Figure 5, Initial conditions $x_{0}=10$ and $s_{0}=90$ :

- Discretization method : 4th order Runge-Kutta

- Discretization step : fix

- Total simulation time : $0.85 \mathrm{~s}$

- Desired convergence tolerance : $1.0000000000 e-010$

- Total CPU in IPOPT : $0.323 s$

- Total CPU in NLP : $0.477 s$

- $\mathrm{Nb}$ of iterations : 31

2. Simulations of Figure 6, medium figures, Initial conditions $x_{0}=10$ and $s_{0}=$ 10 :

- Discretization method : 1st order Explicit Euler

- Discretization step : fix

- Total simulation time : $0.57 \mathrm{~s}$

- Desired convergence tolerance : $1.0000000000 e-012$

- Total CPU in IPOPT : $0.29 s$

- Total CPU in NLP : $0.223 s$

- $\mathrm{Nb}$ of iterations : 40

3. Simulations of Figure 6, medium figures, Initial conditions $x_{0}=10$ and $s_{0}=$ 40 :

- Discretization method : 1st order Explicit Euler

- Discretization step : fix

- Total simulation time : $0.64 \mathrm{~s}$

- Desired convergence tolerance : $1.0000000000 e-012$

- Total CPU in IPOPT : $0.34 s$

- Total CPU in NLP : $0.251 s$

- Nb of iterations : 48

4. Simulations of Figure 6, medium figures, Initial conditions $x_{0}=10$ and $s_{0}=$ 70 :

- Discretization method : 1st order Explicit Euler

- Discretization step : fix

- Total simulation time : $0.37 \mathrm{~s}$

- Desired convergence tolerance : $1.0000000000 e-012$

- Total CPU in IPOPT : $0.207 \mathrm{~s}$

- Total CPU in NLP : $0.138 s$

- Nb of iterations : 37

5. Simulations of Figure 6, bottom figures, Initial conditions $x_{0}=10$ and $s_{0}=10$ :

- Discretization method : 4th order Implicit Gauss

- Discretization step : fix

- Total simulation time : $0.58 \mathrm{~s}$

- Desired convergence tolerance : $1.0000000000 e-005$ 
- Total CPU in IPOPT : $0.21 s$

- Total CPU in NLP : $0.315 \mathrm{~s}$

- Nb of iterations : 20

6. Simulations of Figure 6, bottom figures, Initial conditions $x_{0}=10$ and $s_{0}=40$ :

- Discretization method : 1st order Explicit Euler

- Discretization step : fix

- Total simulation time : $0.32 \mathrm{~s}$

- Desired convergence tolerance : $1.0000000000 e-005$

- Total CPU in IPOPT : $0.16 s$

- Total CPU in NLP : $0.11 s$

- Nb of iterations : 21

7. Simulations of Figure 6, bottom figures, Initial conditions $x_{0}=10$ and $s_{0}=70$ :

- Discretization method : 1st order Explicit Euler

- Discretization step : fix

- Total simulation time : $0.37 \mathrm{~s}$

- Desired convergence tolerance : $1.0000000000 e-005$

- Total CPU in IPOPT : $0.18 s$

- Total CPU in NLP : $0.12 s$

- Nb of iterations : 23 